\title{
Effects of Extreme-Priced Products on Consumer Reservation Prices
}

\author{
Aradhna Krishna, Mary Wagner, and Carolyn Yoon \\ University of Michigan \\ Rashmi Adaval \\ The Hong Kong University of Science \& Technology
}

\begin{abstract}
We show that an extremely high-priced product featured among more moderately priced products within a catalog can increase the reservation price for a moderately priced target product as well as the category as a whole. We investigate how this increase is influenced by the degree of relatedness between the extreme-priced product and the target as well as the situational and temporal proximity (contiguity) in their presentation. Consistent with our conceptualization, we find that the presence of an extreme cue leads to greater changes in target reservation price when the extreme-priced referent and target are more related and are contiguously presented. Furthermore, the impact of an extreme-priced product's relatedness on reservation price appears to be greater when the contiguity between the extreme-priced product and the target product is high versus when it is low.
\end{abstract}

Several major companies have recently included extremely high-priced products in their catalogs-some of which in all probability never sell. For example, the Victoria's Secret catalog for the 2004 holiday season included a gem-studded 10 million dollar bra. Another catalog from the same company featured a half million dollar convertible. Similarly, a recent catalog from Williams Sonoma includes a bread maker for over $\$ 1,000$ and Holt Renfrew, an upscale department store in Canada, features an $\$ 11,500$ coat in its catalog. Because extremely high-priced items such as these rarely sell, this practice raises the question of why marketers include them in their catalogs.

We examine how the inclusion of an extremely highpriced item in marketing settings (e.g., catalogs) influences the magnitude as well as the direction of a consumer's reservation price or the maximum price the consumer is willing to pay for a product. If the mere presence of an extremely high-priced product does indeed increase the reservation price of the target category as a whole or a specific product

Correspondence should be addressed to Aradhna Krishna, Stephen M. Ross School of Business, University of Michigan, 701 Tappan St., Ann Arbor, MI 48109-1234.E-mail: aradhna@umich.edu from that category, then this tactic may be used by marketers to extract higher dollar sales from consumers (by shifting them to a higher-priced product or by charging a higher price for the same product).

This article explores how the presence of an extremely high-priced product can impact the reservation price for both a target category (category reservation price) and a specific product (target product reservation price). It further examines how this impact is contingent on two factors: perceived relatedness (i.e., the similarity between the extreme-priced product and target) and the contiguity of the price presentation (i.e., how closely in space and time the extreme-priced product and the target are encountered).

The effects of these variables were conceptualized in terms of the accessibility-diagnosticity formulation proposed by Feldman and Lynch (1988). That is, the relatedness of the extremely high-priced product to the target product is likely to exert its influence on target evaluations through its impact on perceptions of its diagnosticity. Furthermore, the temporal contiguity of the two products (and, therefore, the recency with which the extremely high-priced product has been encountered) influences the accessibility of this product in memory at the time of judgment. An additional determi- 
nant of its accessibility may be the similarity of the contexts in which the two products have been encountered and, therefore, the likelihood that consideration of the situational features present at the time of target judgment prompts retrieval of the context associated with the extreme-priced product (see Gillund \& Shiffrin, 1984). In general, both the diagnosticity and the accessibility of previously acquired knowledge are necessary conditions for this knowledge to be retrieved and used as a basis for judgment (Higgins, Rholes, \& Jones, 1977; see also Feldman \& Lynch, 1988). Correspondingly, we predict that an extreme-priced product's similarity to the target, its temporal contiguity to it, and the similarity of the situation in which it is encountered all operate in combination to influence the likelihood that people will use this product in deciding the price they will pay for the target product and other products of the same general type.

This research is important for several reasons. Although the impact of extreme values on target judgments is well documented in several research paradigms (e.g., anchoring and adjustment, social judgment theory), the conditions in which it occurs remain unclear. That is, when do extreme values have an impact on judgment and when do they not? First, few if any attempts have been made to identify how consumers decide whether a particular piece of information (e.g., an extreme price) is relevant to the decision at hand. For extreme reference prices to have an influence, consumers have to judge how relevant the information is. This requires an assessment of the similarity of the extreme-priced product to the target. In this context, we are concerned with aspects of the product descriptions that influence this assessment. Second, how important is the temporal and situational contiguity between the exposure to the extreme-priced product and the target in influencing a target product's reservation price? The studies reported here attempt to answer these questions.

\section{CONCEPTUAL FRAMEWORK}

In one stream of research that has dealt with a related issue (Lichtenstein \& Bearden, 1989; Urbany, Bearden, \& Weilbaker, 1988), a target product is presented at a reduced price along with a high external reference price that is either implausible or plausible (e.g., "Was $\$ 499.88$. Now available for $\$ 399.99$ !'”). Respondents are then asked to indicate how much they like the deal (i.e., the target product at the reduced price), and it is assumed that the presence of the high reference price makes the deal price appear more attractive. The results of this research have been mixed (Krishna, Briesch, Lehmann, \& Yuan, 2002). Although Lichtenstein and Bearden (1989) and Urbany et al. (1988) found that implausible reference prices have a significantly positive effect on deal evaluation, Low and Lichtenstein (1993) suggested that implausible deals may lead to negative deal evaluation. Furthermore, Biswas (1992) and Biswas and Blair (1991) found that implausible deals are evaluated as better only for unfa- miliar brands. In general, this stream of research looks at how the extreme prices influence the perception of the deal itself, rather than examining the effect of these extreme prices on the evaluation of other target products or deals. Our research has a somewhat different focus in that it seeks to understand how an extreme price for a product affects the reservation price for targets that belong to other categories or specific targets from the same category.

A second line of research that is more related to the issues that we are concerned with is stimulated by Tversky and Kahneman's (1974) conceptualization of anchoring and adjustment. This stream of research suggests that external anchors have an impact on estimates because respondents use them as a starting point and then adjust up or down the scale. Because they adjust insufficiently, the responses are skewed in the direction of the anchor. Such effects have been demonstrated in a variety of domains and conditions (see Chapman \& Johnson, 2003, for a summary), and they presumably occur because the anchor either influences the manner in which people use the response scale (i.e., it has an influence at the output stage of processing) or because the anchor elicits anchor-related thoughts at an earlier stage of information processing (Strack \& Mussweiler, 1997). Given that Strack and Mussweiler's findings suggested that several anchoring phenomena might be explained by cognitions activated by the anchor, it seems reasonable to suppose that the impact of these thoughts on judgments will depend on (a) how diagnostic the thoughts elicited by the extreme-priced product (anchor) are in judging the target-a factor that might depend on the degree of relatedness between the two and (b) how accessible in memory are the thoughts elicited by the extremepriced product at the time the target judgment is made-a factor that might depend on how contiguous their presentations have been. The effects of these factors are discussed in turn.

\section{How Diagnostic is the Extreme Price?}

The diagnosticity, or applicability, of an extreme-priced product is presumably a function of its relatedness to the target (e.g., the number of features they have in common). Extremely high-priced products might activate thoughts about other premium products that, once activated, may be considered relevant in judging the target product. Research on anchoring phenomena has suggested that anchors have an influence because they act as suggestions and make more salient the information consistent with the anchor (Chapman \& Johnson, 1994, 2003; Jacowitz \& Kahneman, 1995; Mussweiler \& Strack, 1999, 2000; Strack \& Mussweiler, 1997). This could occur either because the anchor serves as a semantic prime (Strack \& Mussweiler, 1997) or because it triggers a biased information search (Chapman \& Johnson, 1994; Schkade \& Johnson, 1989). In the present context, extremely high-priced products could activate thoughts about other premium products that, once activated, might be used 
as inputs in judging the target. We argue that if these thoughts are judged to be sufficiently diagnostic (because of the degree of relatedness of the extreme-priced product to the target), they may influence the reservation price of the target product.

H1: The effect of an extreme-priced product on judgments of a target's reservation price will be greater when it is highly related to the target than when it is not.

Our conceptualization of relatedness is based on three criteria: (a) perceived fit between the product categories to which the extreme-priced product and target belong, (b) the number of shared nonbinary features (e.g., maximum speed of 50,70 , or $90 \mathrm{mph}$ ) between the target and extreme-priced product, and (c) the number of shared binary (yes-no) features (e.g., video camera comes with a carrying case or not) between the target and the extreme-priced product. The first criterion for conceptualizing relatedness is similar to the notion of "fit" that has been advanced in the brand extension literature where extensions fail because they are perceived as incongruent with the parent brand (e.g., mouthwash by Bausch \& Lomb; see Ahluwalia \& Gurhan-Canli, 2000; Keller, 1998). The other two criteria are consistent with the feature similarity model of Tversky (1977) in which the relatedness between the target and the extreme-priced product depends on the number of common and distinctive features that the two share. A binary (yes-no) feature is considered more diagnostic for judging similarities and differences between objects than a nonbinary feature because it has only two values and, therefore, suggests that a product either has the feature or does not. Thus, if there are two products and one has the binary feature and the other one does not, they may be assumed to be very different with respect to this characteristic. On the other hand, differences in nonbinary features are more difficult to assess. Thus, we expect differences in binary (yes-no) features to make differences between the target and extreme-priced product more salient than differences in nonbinary features.

For all three notions of relatedness, we expect that an extreme-priced product that is more related to the target will be perceived to be more useful as an input and hence more diagnostic for making judgments of the reservation price of the target. Although we have no specific predictions for the effects of these different types of relatedness on consumers' reservation prices, we nevertheless examined them empirically in this research to increase the generalizability of our results.

\section{How Accessible Is the Extreme Price?}

The closer the temporal and spatial proximity between exposure to the extreme-priced product and judgment of the target, the more accessible will be the extreme-priced product when consumers evaluate the target's reservation price, and the more likely it is that the extreme price will influence this evaluation. In this context, it is worth noting that Mussweiler and Strack (1999) showed that anchor prices activate anchor-related thoughts. Participants who were asked to consider if the price of a German automobile was greater or less than a high (or low) price later responded more quickly to exemplars of luxury automobiles in the first case and to exemplars of cheaper automobiles in the second case. This finding suggests that an extreme-priced product might not only activate thoughts about similar types of products but that the temporal proximity of exposure to the anchor and question might make these thoughts more or less accessible at the time the target product is considered.

In the context of catalog shopping, extreme-priced products might be presented either on the same page of a catalog as the target product, on a different page, or in a totally different context (a different catalog or in a different shopping situation altogether). The temporal distance between the two products, and the similarity of the situations in which they are encountered, can both produce differences in the accessibility of the extreme-priced product in memory at the time the target is considered. Thus, we propose that the higher the contiguity between the target and the extreme-priced product (the referent), the higher will be the accessibility of the extreme price and the greater its impact on the target's reservation price. More formally, we propose that:

H2: The effect of an extreme-priced product on judgments of a target's reservation price will be greater when it is spatially and temporally contiguous with the target than when it is not.

\section{The Combined Effects of Accessibility and Diagnosticity}

To reiterate, the relatedness of the extreme-priced product to a target (and therefore its diagnosticity) is determined by the similarity of its features to those of the target. The accessibility of this extreme-priced product in memory is a function of its spatial and temporal proximity to the target at the time the target is considered. The effects of these factors on the likelihood of retrieving and using the extreme-priced product as a basis for judgment can be conceptualized in terms of research and theory on knowledge accessibility (Higgins, 1996; Wyer, 2004; Wyer \& Srull, 1989). According to these conceptualizations, the likelihood that thoughts about Concept A (the target) stimulate the retrieval of Concept B (extreme-priced product) is a function of (a) the similarity of Concept B's features to Concept A's (Collins \& Loftus, 1975; Wyer, 2004), (b) the recency with which Concept B was last thought about (Collins \& Loftus, 1975; Higgins, 1996), and (c) the similarity of the situational context in which Concept A is thought about to those in which Concept B was encountered (Gillund \& Shiffrin, 1984). 
It seems reasonable to suppose that an extreme-priced product is unlikely to affect estimates of a target's reservation price if it is not related to the target, or has few features in common with the target. Even if it is related, however, it may not be identified and used as a basis for judgment unless it has been considered only a short time prior to thinking about the target and was encountered in a similar situational context.

H3: The effect of an extreme-priced product's relatedness on a target's reservation price will be greater when its contiguity to the target is high than when it is low.

These hypotheses are tested in three experiments. In Experiment 1 , we considered the extent to which the reservation price of a particular type of product would be influenced by extreme-price anchors pertaining to products varying in their similarity to the target and, therefore, their diagnosticity. The effects of the anchors' temporal contiguity, and the similarity of the context in which they were encountered, were also evaluated. Experiment 2 was similar except that the relatedness between the target and the extreme-priced product was manipulated by varying the number of nonbinary features that were common to the target and the anchor. Furthermore, judgments pertained to a specific target product rather than to a general type of product, as in Experiment 1. A third experiment then sought to confirm these findings under conditions in which the features of the target and anchor were binary.

In the first experiment, the dependent variable is category reservation price. In Experiments 2 and 3, the dependent variable is target product reservation price. We expect that category reservation price would connote the maximum that a person would spend for a product within a category. However, target product reservation price would be for a specific product in that category, and can be considerably lower than the category reservation price depending on the target's perceived quality. We expect $\mathrm{H} 1-\mathrm{H} 3$ to apply to both types of reservation price.

\section{EXPERIMENT 1}

\section{Design}

Experiment 1 examined the effects of contiguity and relatedness on consumers' reservation price. We varied the relatedness of the extreme-priced product to the target over three levels. In addition, we varied the situational context in which the extreme-priced anchor product was encountered, and thus the time interval between exposure to the anchor and presentation of the target. The experiment was a 2 (Contiguity: Low or High) $\times 4$ [Relatedness: Strongly Related (from the same category), Moderately Related (from related categories), Unrelated (from unrelated categories), or Control Group] between-group factorial design.

The two control group participants were not exposed to any extreme price. Thus, there was no conceptual difference between the controls in the two contiguity conditions, and as expected, there were no significant differences in the reservation price for the target product category between the two control conditions $(F<1)$. However, we still included two such conditions purely for the ease of analysis afforded by a fully crossed design.

A total of 162 undergraduate students participated in the study for course credit. They were run in groups ranging from 6 to 8 and were distributed randomly across the study conditions.

\section{Stimuli}

Camera was chosen as the target product category for this experiment. This was chosen on the basis of a pretest with 30 respondents who rated how familiar they were with various product categories and their expertise in making judgments about them on a 9-point scale ranging from 1 (completely unfamiliar) to 9 (completely familiar). The same participants also rated their expertise in making judgments about them on a 9-point scale ranging from 1 (novice) to 9 (expert). Cameras received a moderate familiarity score $(M=5.44)$ and a moderate expertise score $(M=4.53)$.

To determine which product categories would be appropriate for use to manipulate relatedness (diagnosticity), we conducted two additional pretests. In one pretest, 14 participants were asked to rate the relatedness of cameras to various other products on a 7-point scale ranging from 1 (not at all) to 7 (completely). Binoculars were rated as being moderately related $(M=5.07)$ and pens were rated as being relatively unrelated $(M=2.00)$. These mean ratings were significantly different from each other, $F(1,13)=52.12, p<$ .001 . In the second pretest, we assessed the similarity between the moderately related and unrelated products with the target category of cameras. A total of 21 participants rated pens and binoculars on seven 7-point scale items (similarity, fit with company, logical, consistency, representativeness, typicality, and appropriateness) adapted from similarity ratings used in two brand extension studies (Ahluwalia \& Gurhan-Canli, 2000; Taylor \& Bearden, 2002). The scores were averaged across the seven items separately for pens and binoculars. Pens received an average score of 1.72 on the seven ratings, with a reliability of .90 , whereas binoculars received an average scale of 4.42 with a reliability of .96 . These means were significantly different, $F(1,20)=100.74, p<.001$.

In another pretest with 14 participants, we ascertained whether the three levels of target-referent relatedness corresponded to different perceptions of diagnosticity. Participants rated how useful they thought the description of each of the extreme-priced products was in evaluating the target camera on a 9-point scale ranging from 1 (not at all) to 9 (extremely). The strongly related cue (camera) received a mean rating of 6.15 , binoculars received a moderate rating $(M=$ $4.40)$, and pens had a lower rating $(M=1.30)$. The 
diagnosticity ratings were significantly different across products: camera versus binoculars, $F(1,13)=19.98, p<$ .001 ; camera versus pen, $F(1,13)=176.89, p<.001$; and binoculars versus pen, $F(1,13)=49.42, p<.001$.

Finally, in a fifth pretest, which assessed whether the prices of the extreme-priced referent products were indeed perceived as high but plausible, we asked a different set of 16 respondents to rate the plausibility of the extreme price (\$600) for a camera, binoculars, and pen on a 7-point believability scale that ranged from 1 (unbelievably low) to 7 (unbelievably high). Participants also rated the believability of a price of $\$ 60$ for the same three items. The extreme-priced products received mean believability ratings of 6.10 for cameras, 6.07 for binoculars, and 6.14 for pens (no significant differences among the three, $F \mathrm{~s}<1$ ). All seven points on the scale had descriptive anchors and 6 was labeled as high, but believable. We thus interpreted these mean ratings as indication that most respondent found the extreme price to be high but plausible, and only a few individuals found it to be unbelievably high. By comparison, the typically priced cameras (price of $\$ 60$ ) received a mean rating of 3.71 on the same believability scale with 3 corresponding to slightly low and 4 about the right price.

\section{Procedure}

On arrival to the experimental session, participants were told that they were taking part in a catalog study and that they would be shown some pages taken from a catalog for products soon to be introduced by a (hypothetical) company. They were informed that the pages represented a rough copy version of a catalog still being designed and tested. Each catalog featured color pictures of the products with a brief description of product characteristics, including price. The products in the catalog appeared on separate pages and were assigned letter and number combinations. To lend greater realism to the cover story, an order form was included in each catalog, although participants were told that they did not have to fill it out. The experimenter informed participants that they would be asked some questions related to the catalog after they had viewed it at their own pace.

In each of the eight conditions, participants saw a cata$\log$ containing eight different typically priced target cameras $(M=\$ 60$, range $=\$ 50-\$ 75)$. In addition, all participants, with the exception of those in the control conditions, saw an extreme-priced product from one of the following product categories: cameras (strongly related), binoculars (moderately related), or pens (unrelated). Thus, the control condition had eight products, whereas the experimental conditions had nine. This confound was eliminated in the two subsequent experiments. These extreme-priced products were priced at $\$ 600$, or approximately 10 times the average price of the cameras in the catalog. As reported previously, this amount was assessed through pretests to be extreme but not implausible. Prices of the extreme-priced products remained constant across conditions-this was done to avoid potentially confounding product effects with extreme-price effects.

In the high contiguity condition, the extreme-priced product appeared in the same catalog on the fifth page (nine pages in total). In the low contiguity condition, the extreme-priced product was presented separately and prior to the introduction of the catalog, in a seemingly unrelated task. The participants in this condition were told that they were to complete several different studies within the experimental session. For the first "study," they were given a separate page containing the extreme-priced product, and were asked to consider their general impressions of the product. They were told that the short study was intended to put them in a "relaxed but thinking mode." The single pages were then taken away from the participants by the experimenter. This was followed by the administration of the second study in the session, comprising a filler task lasting approximately $15 \mathrm{~min}$. Those assigned to the high contiguity condition did not engage in the first "study" task and started with the filler task.

Study participants were allowed to examine the catalog as they filled out the main questionnaire containing the dependent measures (in the low contiguity condition, participants could not see the extreme-priced product while filling out the questionnaire). Participants were asked to write down the maximum price they were willing to pay for the target product category (cameras). This was determined by each participant's response as to the maximum amount of money he or she would be willing to pay for a camera (i.e., category reservation price). Participants were then asked to write down any thoughts that came to mind as they viewed the catalog. On completion of the questionnaire, they were debriefed, thanked for their participation, and dismissed.

\section{Results}

Category reservation prices are shown in Table 1 as a function of target-referent contiguity and relatedness. The maximum price that participants were willing to pay for a product in the target category varied as expected with how related it was to the extreme-priced referent product $(M \mathrm{~s}=\$ 174.12$,

TABLE 1

Experiment 1 Results: Category Reservation Price by Target-Referent Contiguity and Relatedness

\begin{tabular}{lcc}
\hline & \multicolumn{2}{c}{$\begin{array}{c}\text { Target-Referent } \\
\text { Contiguity }\end{array}$} \\
\cline { 2 - 3 } Target-Referent Relatedness & Low & High \\
\hline Control group (no referent) & $119.00_{\mathrm{a}}$ & $115.75_{\mathrm{a}}$ \\
Unrelated referent (pen) & $94.00_{\mathrm{a}}$ & $163.16_{\mathrm{c}}$ \\
Moderately-related referent (binoculars) & $100.75_{\mathrm{a}}$ & $186.82_{\mathrm{c}}$ \\
Strongly-related referent (camera) & $154.14_{\mathrm{b}}$ & $197.11_{\mathrm{c}}$ \\
\hline
\end{tabular}

Note. Mean difference scores in the same column that do not share subscripts differ at $p<.05$. 
$\$ 143.78, \$ 128.58$, and $\$ 117.38$ under strongly related; moderately related; unrelated; and control conditions, respectively), $F(3,154)=3.94, p<.01$. More detailed analyses revealed that the difference in the reservation price estimates between the treatment and control conditions was significant when the extreme-priced product and target were strongly related, $F(1,154)=8.49, p<.005$, and marginally significant when moderately related, $F(1,154)=2.75, p<.10$. However, the reservation prices in the unrelated and control conditions were not significantly different $(F<1)$. This pattern of results supports $\mathrm{H} 1$.

Participants generated higher estimates of the price they would pay for a camera if they had been exposed to the extreme-priced product in close proximity to the target category $(M=\$ 165.71)$ than if they had encountered it in a different task $(M=\$ 116.97), F(1,154)=15.93, p<.0001$. Thus, as implied by $\mathrm{H} 2$, reservation prices were influenced by the contiguity of the extreme-priced product.

The main effects of contiguity and relatedness were, however, qualified by a significant (marginal) interaction between them, $F(3,154)=2.53, p<.06$. In the strong relatedness condition, category reservation price was significantly higher relative to the control condition both under high contiguity, $F(1,154)=10.38, p<.004$, and under low contiguity, $F_{\text {dir }}(1,154)=2.99, p<.05$. However, in the moderately related and unrelated conditions, category reservation price was significantly higher than the control only under high contiguity for moderately related, $F(1,154)=8.52, p<.006$, and for unrelated, $F(1,154)=3.52, p<.02$; but not under low contiguity conditions $(F \mathrm{~s}<1)$. Thus, we obtained some support for $\mathrm{H} 3$ with the data indicating that the effect of relatedness was generally more evident in the high contiguity conditions than in the low contiguity conditions.

\section{Mediation Analysis}

Our conceptual framework suggests that accessibility of the extreme price mediates the effects of contiguity on category reservation price. Accessibility of the extreme price was measured by coding participants' cognitive responses for the number of extreme price-related thoughts. That is, participants' written protocols were coded in terms of thoughts focusing on the extreme price and those not focusing on the price, by two independent judges who were blind to the study treatments and hypotheses. Interrater reliabilities across the scored items were greater than .87 , with scoring differences resolved via discussion.

We tested for mediation using the procedure suggested by Baron and Kenny (1986). First, the analysis of category reservation price (dependent variable) as a function of contiguity (predictor) shows that the latter has a significant effect $(\beta$ $=46.71, p<.01$ ); thus the manipulated factor affects the dependent variable in a regression that does not contain the mediator. Second, the analysis of accessibility measured by the number of price-related thoughts (mediator) as a function of contiguity shows that the latter has a significant effect $(\beta=$ $.38, p<.01)$; hence the predictor affects the mediator. Third, the analysis of reservation price (dependent variable) as a function of both contiguity and accessibility shows only the accessibility effect-accessibility was a significant predictor $(\beta=116.24, p<.001)$ and contiguity was not $(\beta=2.76, p>$ $.70)$. Thus, the mediator affects the dependent variable, but the predictor effect vanishes. This established full mediation and supports our contention that cue accessibility mediates the impact that target-extreme cue contiguity has on target category reservation price.

\section{Discussion}

This study examined how extreme prices from product categories that are strongly related, moderately related, or unrelated to the target category affect the direction and magnitude of consumer reservation prices. It further investigated how these results are affected by the contiguity of the extreme-priced product and the target. Overall, strong relatedness of the extreme-priced cue and target resulted in significantly higher category reservation prices regardless of whether the extreme cue was seen in the same catalog as the target (high contiguity) or separate from it (low contiguity). When the extreme cue was moderately related or unrelated to the target, it had an impact on category reservation price only when contiguity was high. Process-level data validate the claim that accessibility mediates the effect that contiguity has on category reservation price.

Note that besides "relatedness," cameras, binoculars, and pens differ from each other in other respects as well, such as complexity and familiarity (binoculars arguably being the most complex or least familiar product). However, the extreme prices we used for the three products were the same (\$600) and yielded results consistent with our explanations. Although we cannot rule out within this study the possibility that these other dimensions may moderate the extreme-price effects, we assert that accounting for them is unlikely to advance our theoretical understanding of the nature of relations among the critical variables of interest.

\section{EXPERIMENT 2}

Experiment 2 was similar to Experiment 1 with three exceptions. First, we assessed reservation prices of a (specific) target product rather than for a general product category. Second, we varied relatedness within a given product category rather than across categories. Specifically, the extreme-priced product and the target belonged to the same product category. However, the overlap in product-based features between the extreme-priced product and target product was varied over three levels. Finally, we varied the price (extreme vs. nonextreme) of the product that served as a referent for the target. Thus, Experiment 2 eliminated a confound 
present in Experiment 1 in which the treatment conditions always contained an additional product (the extreme-priced referent) compared to the control condition. In Experiment 2, we included a separate control for each experimental condition-a regularly priced product condition that was identical to the extreme-priced product along all dimensions other than price (the nonextreme priced-referent). The difference in reservation price estimates when people were exposed to a nonextreme-priced referent and an extreme-priced referent thereby provided an indication of whether the extreme reference prices had any influence on reservation prices.

\section{Pretests}

Hybrid bicycles were chosen as the product category featured in the catalog in Experiment 2. This category has product attributes with which most participants are relatively unfamiliar. Therefore, the same product could be assigned different prices (reference price: extreme vs. nonextreme) without the specific attributes alerting participants as to which price is more appropriate for the product. The product was priced moderately at $\$ 375$ or extremely at $\$ 3,725$. These prices were pretested with 14 participants on the same 7-point scale used in the pretest for Experiment 1. Based on the pretest, the moderate price was considered about right $(M$ $=3.64$ ), and the extreme price was considered high but plausible $(M=6.21)$. These means were significantly different, $F(1,13)=89.68, p<.001$.

Another pretest with 77 participants was also conducted to check if the levels of relatedness between the target and the referent made the price cue more or less diagnostic. Using the same 9-point scale described in the pretest for Experiment 1, the price cue of the product with five shared nonbinary features (high relatedness) was perceived to be the most diagnostic $(M=8.24)$, followed by the one with three shared nonbinary features (moderate relatedness; $M=6.88$ ), and then zero shared nonbinary features (low relatedness; $M=$ 4.69). Significant differences were found for all three pairwise comparisons: zero versus three shared, $F(1,74)=$ $23.62, p<.001$; zero versus five shared, $F(1,74)=56.70, p<$ .001 ; and three versus five shared, $F(1,74)=12.43, p<.01$.

\section{Design, Stimuli, and Procedure}

Experiment 2 was a 2 (Reference Price: Extreme or Nonextreme) $\times 2$ (Target-Referent Contiguity: Low or High) $\times 3$ (Target-Referent Relatedness: Low $[0=$ shared nonbinary features], Moderate $(3=$ shared nonbinary features], or High [ $5=$ shared nonbinary features]) between-group factorial design. The dependent variable was the amount people were willing to pay for a specific product (i.e., target product reservation price). We did not elicit the category reservation price because of the potential confounding effect of eliciting both reservation price for the product and the category from the same participant.
A total of 197 students in a large university participated in the experiment in groups ranging from 6 to 12 . They were distributed randomly across the 12 conditions. The experimental procedure was similar to Experiment 1 . Thus, participants in the low contiguity condition viewed the extreme or nonextreme-priced referent product separately, in a seemingly unrelated task, prior to the introduction of the catalog containing eight products (the reader may recall that in Experiment 1 , there was no nonextreme referent). By contrast, those in the high contiguity condition received a catalog containing all nine products including the target product and either the extreme or nonextreme-priced referent product. Product descriptions contained color pictures of the products and a brief description of five product features (all of which were nonbinary features; see Figure 1). The products in the catalog were assigned letter and number combinations for names and appeared on separate pages. The referent product (i.e., the extreme- or nonextreme-priced product) shared zero, three, or five features with the target bicycle. Price information was listed for each bicycle in the catalog, except for the target product, which was accompanied by the statement, "Price to be announced." The target product always appeared fifth in the catalog (in high [low] contiguity conditions with nine [eight] products in the catalog). A total of 12 catalogs were created-1 for each treatment condition. The order of the products in the catalog was not varied.

Participants in all conditions were allowed to examine the catalog while completing the questionnaire. However, note that in the low contiguity condition, participants could not see the extreme cue while filling out the questionnaire. Each participant wrote down the maximum amount he or she would be willing to pay for the target bicycle that was held constant across all conditions and appeared with the statement, "Price to be announced." The catalog and questionnaire were then taken away and the participant completed a short unrelated filler task. Then individuals were given the second part of the questionnaire in which they wrote down any thoughts that had come to mind as they viewed the catalog. On completion of the questionnaire, they were debriefed, thanked for their participation, and dismissed.

\section{Results}

Analysis of variance procedures were conducted using target reservation price as the dependent variable, and reference price (extreme vs. nonextreme), contiguity (high vs. low), and relatedness (high, moderate, or low) as the independent variables.

Reservation prices are summarized in Table 2 as a function of reference price, contiguity, and relatedness. The impact that the extreme-priced product had in each treatment condition is indicated by the difference between the target reservation price when the reference price was extreme versus when it was not $\left(M_{\text {diff }}\right)$. These effects are shown in Columns 3 and 6 of Table 2. 
Target Product

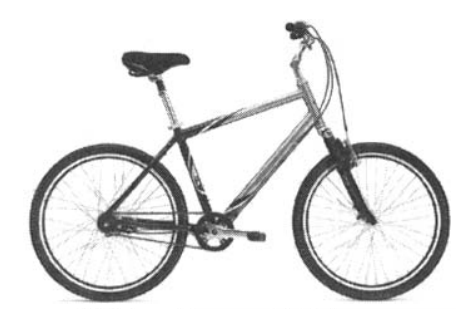

Low Target-Referent Relatedness

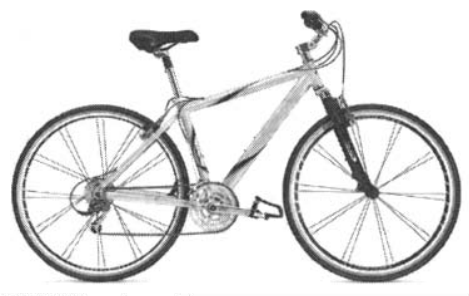

\begin{tabular}{|l|l|}
\hline Item: WL-6654 & Item: E0-7512 (N0-7512) \\
\hline Pedals: Dual density platform & Pedals: Consistent density platform \\
\hline $\begin{array}{l}\text { Brakes: Max with linear pull front with } \\
\text { modular, rear roller with cooling disc }\end{array}$ & $\begin{array}{l}\text { Brake: AVID M420 with modulator on front } \\
\text { with two-axis alloy levers }\end{array}$ \\
\hline $\begin{array}{l}\text { Wheel Set: 750 rims with 7 speed Nexxon } \\
\text { Rear and alloy quick release front hubs; 14G } \\
\text { stainless spokes }\end{array}$ & $\begin{array}{l}\text { Wheel Set: Paired Spoke Technology; torque } \\
\text { sharing super-stiff rear hub; semi-deep front } \\
\text { rim; double butted 14/15 DT spokes }\end{array}$ \\
\hline Saddle: Cyclex Webspring Comfort & Saddle: Cyclex Cushion CRT \\
\hline Frame: Alpha Aluminum Comfort Specific & $\begin{array}{l}\text { Frame: Alpha Super Light Aluminum, with bi- } \\
\text { axial downtube and computer butted head tube }\end{array}$ \\
\hline Price: To Be Announced & Price: \$3,725 (\$375) \\
\hline
\end{tabular}

Moderate Target-Referent Relatedness (3-Shared Nonbinary Features)

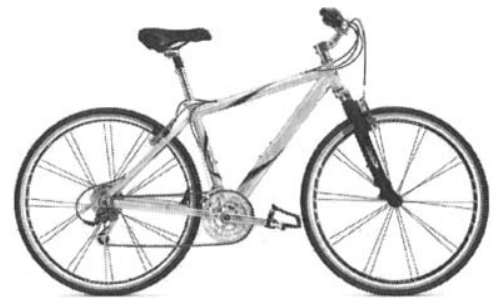

High Target-Referent Relatedness (5-Shared Nonbinary Features)

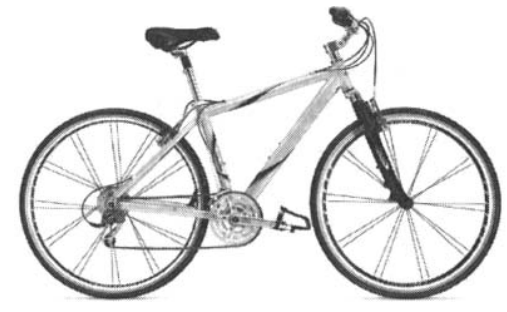

\begin{tabular}{|l|l|}
\hline Item: E3-7512 (N3-7512) & Item: E5-7512 (N5-7512) \\
\hline Pedals: Dual density platform & Pedals: Dual density platform \\
\hline $\begin{array}{l}\text { Brake: Max with linear pull front with } \\
\text { modular, rear roller with cooling disc }\end{array}$ & $\begin{array}{l}\text { Brake: Max with linear pull front with } \\
\text { modular, rear roller with cooling disc }\end{array}$ \\
\hline $\begin{array}{l}\text { Wheel Set: 750 rims with 7 speed Nexxon } \\
\text { Rear and alloy quick release front hubs; 14G } \\
\text { stainless spokes }\end{array}$ & $\begin{array}{l}\text { Wheel Set: 750 rims with 7 speed Nexxon } \\
\text { Rear and alloy quick release front hubs; 14G } \\
\text { stainless spokes }\end{array}$ \\
\hline Saddle: Cyclex Cushion CRT & Saddle: Cyclex Webspring Comfort \\
\hline $\begin{array}{l}\text { Frame: Alpha Super Light Aluminum, with bi- } \\
\text { axial downtube and computer butted head tube }\end{array}$ & Frame: Alpha Aluminum Comfort Specific \\
\hline Price: $\mathbf{\$ 3 , 7 2 5} \mathbf{( \$ 3 7 5 )}$ & Price: $\mathbf{\$ 3 , 7 2 5} \mathbf{( \$ 3 7 5 )}$ \\
\hline
\end{tabular}

FIGURE 1 Examples of hybrid bike stimuli in Experiment 2. 
TABLE 2

Experiment 2: Reservation Price by Reference Price, Target-Referent Contiguity, and Relatedness

\begin{tabular}{|c|c|c|c|c|c|c|}
\hline \multirow[b]{2}{*}{ Target-Referent Relatedness } & \multicolumn{3}{|c|}{ Low Target-Referent Contiguity } & \multicolumn{3}{|c|}{ High Target-Referent Contiguity } \\
\hline & $\begin{array}{c}\text { Extreme } \\
\text { Price }\end{array}$ & $\begin{array}{c}\text { Nonextreme } \\
\text { Price }\end{array}$ & $\begin{array}{c}\text { Mean } \\
\text { Difference }\end{array}$ & $\begin{array}{c}\text { Extreme } \\
\text { Price }\end{array}$ & $\begin{array}{c}\text { Nonextreme } \\
\text { Price }\end{array}$ & $\begin{array}{c}\text { Mean } \\
\text { Difference }\end{array}$ \\
\hline Low $(0)$ & 392.94 & 357.65 & $35.29 \mathrm{a}$ & 340.31 & 377.50 & $-37.19_{a}$ \\
\hline Moderate (3) & 379.06 & 368.82 & $10.24_{a}$ & 568.24 & 307.81 & $260.42_{c}$ \\
\hline $\operatorname{High}(5)$ & 393.44 & 310.00 & $83.44_{b}$ & 907.19 & 333.08 & $574.11_{d}$ \\
\hline
\end{tabular}

Note. Mean difference scores in the same row and in the same column that do not share subscripts differ at $p<.05$.

As the data show, the effect of the extreme-priced product was greater when contiguity was high $\left(M_{\text {diff }}=265.78\right)$ than when it was low $\left(M_{\text {diff }}=42.99\right), F(1,185)=4.60, p<.05$. Similarly, the effect of the extreme-priced product was greater when the target-referent relatedness was high $\left(M_{\text {diff }}=\right.$ $328.78)$ than when it was moderate $\left(M_{\text {diff }}=135.33\right)$ or low $\left(M_{\text {diff }}=-0.95\right), F(2,185)=3.39, p<.05$. Furthermore, the three-way interaction of Contiguity $\times$ Relatedness $\times$ Reference Price was significant, $F(2,185)=3.99, p<.05$, and is attributable to the fact that the influence of relatedness on the impact of price was substantially greater when contiguity was high than when contiguity was low.

We would, however, like to note that our results for $\mathrm{H} 3$ are driven in part by the fact that the reservation price for the nonextreme controls vary quite a lot (even though there is no theoretical reason for this to occur). If one were to ignore the nonextreme controls and focus merely on reservation prices in the extreme reference price conditions (Columns 1 and 4 of Table 2), the interaction of Contiguity $\times$ Relatedness is significant, $F(1,92)=4.04, p<.05$; also significant are the main effects of contiguity, $F(1,92)=4.38, p<.05$, and relatedness, $F(2,92)=3.54, p<.05$.

\section{Mediation Analysis}

To conduct similar mediation analyses as in Experiment 1, we again treated the number of price-related thoughts generated by participants as a measure of accessibility. Interrater reliabilities across the scored items were greater than .90 , with scoring differences resolved via discussion.

As before, we tested for mediation using the procedure suggested by Baron and Kenny (1986). First, the analysis of target reservation price (dependent variable) as a function of contiguity (predictor) shows that the latter has a significant effect $(\beta=100.76, p<.05)$, indicating that the manipulated factor affects the dependent variable in a regression that does not contain the mediator. Second, the analysis of accessibility measured by the number of price-related thoughts (mediator) as a function of contiguity shows that the latter has an effect $(\beta=.34, p<.001)$; hence the predictor affects the mediator. Finally, the analysis of reservation price (dependent variable) as a function of both contiguity and accessibility shows that accessibility $(\beta=60.03, p<.05)$ was a significant predictor and contiguity was not $(\beta=79.96, p>.17)$; thus, the mediator affects the dependent variable, but the predictor effect vanishes. This again establishes full mediation and supports our contention that cue accessibility mediates the impact that contiguity of the extreme-priced referent to the target has on target reservation price.

\section{Discussion}

The presentation format in Experiment 2 enabled the examination of the effect of the number of shared nonbinary features on reservation price. The results are consistent with our predictions and suggest that extreme reference prices might exert their influence on reservation prices through their effect on contiguity (accessibility) and relatedness (diagnosticity). Furthermore, they suggest that feature similarity might be an interesting variable to examine more closely. In this regard, it is worth noting that although some products in the real world can be described by different levels of the same feature (e.g., "super light aluminum" frame vs. "aluminum" frame), others may be described as either possessing or not possessing a certain feature of a particular product. For example, one pair of sunglasses may be scratch resistant, whereas another pair may not be. This may make differences between products more salient and place greater restrictions on when the extreme-priced product has an effect.

In the next study, we examine how a presentation format describing unique features (rather than shared features) of products from the same category will impact target reservation price-that is, whether target reservation price will still increase with this presentation format for moderate and high relatedness between the target and the extreme-priced product cue. We thereby seek in Experiment 3 to investigate whether there are boundary conditions on the increases in reservation prices when we vary relatedness by manipulating the number of binary (yes-no) features that the target possesses.

\section{EXPERIMENT 3}

As stated earlier, in Experiment 2 the relatedness between the target and the extreme-priced product was manipulated 
by varying the difference in their nonbinary features (Tversky, 1977). In contrast, this variable is manipulated in Experiment 3 by varying the differences in their binary features. We conjectured that a binary (yes-no) feature would be more diagnostic for assessing similarities and differences between the target and the referent compared to nonbinary features. For example, if the referent has the binary feature and the target does not, then the two products will be perceived to be as far apart on this feature as possible. With nonbinary features, this is not the case. Thus, we expect differences in binary (yes-no) features, more so than differences in nonbinary features, to make salient the relatedness between the target and extreme-priced referent product. Experiment 3 examines if our theorizing holds when we vary relatedness (three levels) in the manner described as well as contiguity (three levels).

\section{Pretests}

Treadmills were selected as the product category. They were relatively unfamiliar to participants and therefore, the same product could serve as the extreme-priced and the nonextreme-priced referent product without arousing suspicion. In addition, the choice of this category allowed us to manipulate binary features without participants questioning the realism of the products. We also pretested the low and high prices for treadmills and ensured that they fell within a range perceived as plausible by study participants. Twenty-one participants rated the plausibility of price ranges for treadmills (obtained from the Internet) using the scale described in the previous experiments. A price range of $\$ 450$ to $\$ 500$ received a mean rating of 3.29. A price range of $\$ 4,500$ to $\$ 5,000$ received a mean rating of 6.14 . These ratings were significantly different from each other, $F(1,20)=151.89, p<$ .0001 . Based on these results, $\$ 4,975$ was selected as the extreme reference price and $\$ 500$ as the nonextreme reference price.

Nine different descriptions of the treadmill models were prepared for the experiment. Three of these were to serve as target products and six as nontarget products. The features described in these models were broken down into two types: binary (yes-no) and nonbinary features. The nonbinary features (maximum incline, maximum speed, and runner weight limit) were balanced throughout a set of nine product descriptions to be used in the experiment, so that the products would be evaluated as being approximately equivalent on these nonbinary features. To confirm that the nonbinary features were equivalent, a pretest with 21 participants was conducted and respondents were asked to compare some pairs of the six nontarget products. Participants were presented with the nonbinary feature information for three pairs of products and asked to evaluate the relative quality of each pair on a 7-point scale ranging from 1 (Product $A$ is much better than Product B) to 7 (Product B is much better than Product A). The presentation of the pairs was counterbalanced. The mean ratings for each pair were $4.17,3.76$, and 4.00 . These means were not significantly different $(F \mathrm{~s}<1)$.

Next, five possible binary features (advanced cushioning system, automatic incline, foldable, heart rate sensor, and constant pace display) were selected. The extreme (or nonextreme) referent cue possessed all five, whereas the target products possessed zero, two, or five of these nonbinary features.

Another pretest was conducted to determine whether the levels of relatedness between the target and the referent corresponded to different perceptions of diagnosticity. Using the same 9-point scale as in the pretests for Experiments 1 and 2, participants rated how diagnostic the referent product was. Ratings showed that when the referent product had five shared binary features with the target (high relatedness), it was perceived to be the most diagnostic $(M=7.40)$. This was followed by the referent product that had two shared binary features (moderate relatedness; $M=5.62$ ) and the one with zero shared binary features (low relatedness; $M=4.19$ ). Significant differences emerged for paired comparisons of each of these means: for five versus zero shared, $F(1,24)=$ $52.99, p<.001$; five versus two shared, $F(1,24)=25.60, p<$ .001 ; and two versus zero shared, $F(1,25)=8.94, p<.01$.

\section{Design, Stimuli, and Procedure}

A total of 72 individuals participated in groups ranging from 6 to 12. Each participant received information about three target products. One half of these participants received this information in the context of an extreme-priced referent and the other one half in the context of a nonextreme-priced referent (a between-group manipulation of reference price). The three target products each represented a different combination of relatedness (high, moderate, and low) and contiguity (high, moderate, and low). These combinations varied over participants in a Greco-Latin square design, as indicated. In choosing the study design, some difficult trade-offs were made. Although the selected design precluded the direct testing of interactions involving Relatedness $\times$ Contiguity, it allowed an economy of experimental effort in assessing $\mathrm{H} 1$ and $\mathrm{H} 2$. The design also afforded potentially useful insights with respect to $\mathrm{H} 3$ via an inspection of the data patterns (for a discussion of the schematic representation our design, see Plan 13 in Winer, 1971, pp. 748-749).

Each respondent saw nine different descriptions of treadmills. The extreme (nonextreme) priced referent always appeared in the third position in the catalog. Two of the three target products were also presented in the catalog and the third target product was presented on the separate sheet of paper that respondents saw after they had viewed the other items in the catalog (this served as our low contiguity manipulation and will be discussed shortly). The remaining five products served as fillers. Of the nine treadmills viewed, the five fillers were moderately priced, the referent was either priced high or moderate, and the remaining three treadmills 
that served as targets were not priced and carried a statement, "Price to be announced."

To manipulate contiguity of the targets relative to the referent product, we presented two of the three targets and the referent in the same catalog, and the third target separately. This lent greater realism to the study by simulating the conditions in which catalog items might appear. The three target products that respondents saw appeared in one of following locations: across the page from the referent (high contiguity), within the catalog but on the following page (moderate contiguity), and on a separate sheet (low contiguity). Thus, in high contiguity conditions, respondents could compare the extreme (nonextreme) referent to the target directly because they were presented on two adjacent pages. In moderate contiguity conditions, they had to turn the page to make this comparison and in the low contiguity condition, the target was presented after a few other filler product descriptions and was physically separated from the rest.

The three target products that each respondent evaluated varied not only in contiguity to the target but also in its relatedness. This was accomplished using a Greco-Latin square procedure in which each target product $(\mathrm{A}, \mathrm{B}$, and $\mathrm{C}$ - each of which was described by a different picture and nonbinary features) was seen only once by a respondent and was associated with different configurations of relatedness and contiguity. Thus, the first group of respondents saw a catalog containing Target Product $\mathrm{C}$ (high contiguity-low relatedness), followed by Target Product B (moderate contiguity-high relatedness), followed by Target Product A (low contiguity-moderate relatedness). A second group saw Target Product B (high contiguity-moderate relatedness), followed by Target Product A (moderate contiguity-low relatedness), followed by Target Product $\mathrm{C}$ (low contiguity-high relatedness). Finally, a third group saw Target Product A (high contiguity-high relatedness), followed by Target Product $\mathrm{C}$ (moderate contiguity-moderate relatedness), followed by Target Product B (low contiguity-low relatedness). Thus, although participants always saw the high contiguity product first, the moderate contiguity second, and the low contiguity third, product type (pictures plus nonbinary features) and relatedness were counterbalanced within this fixed order. Note that we manipulated contiguity by presenting the products in a catalog and a separate sheet and as a result of this were forced to keep the order of contiguity the same. The realism this catalog presentation procedure afforded us, however, placed further design limitations on this study.

Respondents were introduced to the study with instructions that they would be shown pages taken from a catalog for products that soon would be introduced in the market. Respondents were informed that the catalog was still in the process of being designed. This explained the rough version and provided an explanation for why all pages were not stapled together. In the instructions, respondents were informed that they would be asked several questions regarding the products they were about to view. They were then given the catalog containing the eight product descriptions and after they had examined them, they were given the last model on a separate sheet. After they had been exposed to all nine models, reservation prices for each of the three target products were elicited through a questionnaire that counterbalanced the order in which the questions were asked. These responses served as the main dependent variables.

\section{Results}

In accordance with standard procedure for analyzing the data from an experiment with a Greco-Latin square design, we conducted two sets of analyses of variance with target reservation price serving as the dependent variable. In the first analysis, reference price (extreme vs. nonextreme) and group $(1,2$, or 3$)$ were between-subject factors and relatedness (low, moderate, or high) was a within-subjects factor. In the second, reference price and group were between-subject factors, and contiguity (low, moderate, or high) was a within-subjects factor. Although the interaction effect of Contiguity $\times$ Relatedness (H3) could not be assessed statistically given the Greco-Latin square design, an evaluation of the effect of relatedness at each level of contiguity was nonetheless beneficial and will be discussed.

The first analysis yielded a significant effect of reference price, $F(1,64)=14.45, p<.001$; a significant effect of relatedness, $F(2,128)=27.13, p<.001$; and an interaction of Reference Price $\times$ Relatedness, $F(2,128)=18.96, p<.001$. These effects were not contingent on groups $(F \mathrm{~s}<1)$. As can be seen from the data in Table 3 , the effect of reference prices was substantially greater when target-referent relatedness was high $\left(M_{\text {diff }}=927.58\right)$ than when it was either moderate $\left(M_{\text {diff }}=66.86\right)$ or low $\left(M_{\text {diff }}=-3.99\right)$, and this was true regardless of the contiguity between exposure to the reference price and exposure to the target. This pattern of results is consistent with $\mathrm{H} 1$.

The second analysis treated the contiguity as a within-subjects factor as noted earlier and tested $\mathrm{H} 2$. According to $\mathrm{H} 2$, the effect of reference price should be greater when contiguity is high than when it is low. Although neither the main effect of contiguity nor its interaction with reference price was significant $\left(F_{\mathrm{S}}<1\right)$, we obtained a significant interaction of Contiguity $\times$ Reference Price $\times$ Group, $F(4,128)=$ $9.96, p<.001$. Further inspection of this interaction shows that it was largely driven by mean differences between extreme versus nonextreme reference prices that were represented in different cells within each group level. It was therefore not informative and the analysis clearly showed that $\mathrm{H} 2$ was not supported in this experiment.

As noted earlier, although the interaction effect of Contiguity $\times$ Relatedness (H3) could not be estimated, the data in Table 3 show that the predicted effect of contiguity appeared to be restricted to conditions in which target-referent relatedness was high and was not at all evident at lower levels of relatedness. This contingency is, in fact, consistent with H3. 


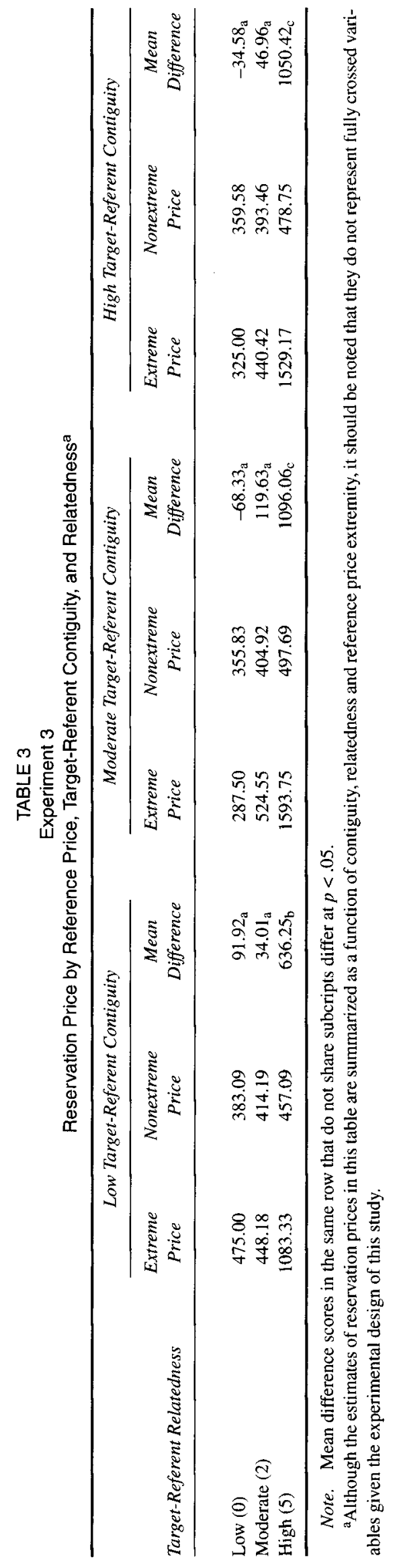


According to this hypothesis, the impact of relatedness should be greater when contiguity is high than when it is low. The results show that the mean difference in the effect of reference price in the high versus low relatedness conditions is greater when contiguity is high (1050.42 vs. -34.58$)$ than when it is low (636.25 vs. 91.92). The source of this difference is clearly localized in the high relatedness condition. That is, the effect of the reference price in this condition was substantially greater when contiguity was high or moderate than when it was low.

\section{Discussion}

Experiment 3 showed that the extreme price influenced target reservation price to a greater extent when there was a high degree of relatedness between the two products $(\mathrm{H1})$. Although the effect of this extreme price was not greater when contiguity between the referent and the target was high than when it was low (H2), the effect of relatedness did appear to depend on contiguity (H3). The data pattern clearly suggests that the impact of the extreme-priced reference product is greater when its relatedness to the target products is high versus low, particularly so in high and moderate contiguity compared to low contiguity conditions.

The absence of an overall effect of contiguity on the influence of reference price (H2) is somewhat unexpected. It is conceivable that when the features used to characterize the target and the referent are dichotomous (binary features) rather than continuous, they make referent products appear more dissimilar (less related) and less diagnostic in estimating the target's reservation price. In these cases, a cursory observation by the respondents might be sufficient for them to realize that the extreme-priced product is not useful in judging the target even if they are presented close to each other. This might account for the lack of a significant overall effect of contiguity.

The one qualification that must be noted in this context is that when the two products shared a large number of binary features (high relatedness condition), the extreme-priced product had a greater effect in high and moderate contiguity conditions $\left(M_{\text {diff }}=1050.42\right.$ and 1096.06$)$ relative to low contiguity conditions $\left(M_{\text {diff }}=636.25\right)$. This data pattern is consistent with $\mathrm{H} 3$ in that it suggests that in the more proximal conditions, participants might have noticed the similarity between the products. The information about the features might have stayed in memory leading to bigger effects when a similar target was encountered shortly afterwards. Although we did not assess memory for product features or measure accessibility in the different conditions, it seems reasonable to suppose that two highly overlapping attribute sets will be recalled more than two sets that are nonoverlapping. Furthermore, accessibility should be greater in the former case than in the latter.

We wish to point out one other potential ambiguity in $\mathrm{Ex}-$ periment 3 . The greater the number of binary attributes, the higher the perceived quality of the product may be, because presumably the presence of any additional feature is "good." Hence, the more related the target product is to the referent, the higher the reservation price relative to conditions in which it is not that highly related. However, given the results of Experiments 1 and 2, it is unlikely that the very high reservation price of approximately $\$ 1,500$ in the high relatedness condition can be explained merely by the "goodness" of additional binary features. Rather, it is more likely the case that reservation price is driven to be so high because of the contiguity of the $\$ 4,975$ extreme-priced referent.

In conclusion, when the target and extreme referent products were moderately related or unrelated, contiguity of the extreme cue did not exert a differential influence on participants' formulation of their reservation price for the target. It seems that differences in binary features make the target and referent product look very dissimilar, so that the extreme cue is ignored even when accessible. However, when the target and extreme-priced product are highly related and described by binary features, contiguity appears to exert some influence on the impact of extreme reference prices.

\section{GENERAL DISCUSSION}

The three studies in this article, taken together, show clearly that extreme-priced products can influence the maximum amount consumers are willing to pay for a product category and specific target products. This result is quite disturbing from a public policy perspective because it implies that one extreme cue can impact the reservation price for many products (and many product categories, going by the results of Experiment 1). Although this basic influence has been documented elsewhere (see Nunes \& Boatwright, 2004), our findings provide further insight into the processes that underlie these effects. Although the studies by no means provide a comprehensive picture of when such effects are likely to occur, they do outline certain basic conditions that need to be met before the extreme-priced product exerts an influence on consumers' reservation prices.

Our conceptualization of these findings is based on the accessibility-diagnosticity framework proposed by Feldman and Lynch (1988) and memory models developed earlier by Collins and Loftus (1975), Gillund and Shiffrin (1984), and Wyer and Srull (1989). Our conceptualization suggests that the interrelatedness between various product categories will hold the key to understanding reference price effects. If a consumer sees an extreme-priced product, considers it outrageously priced and walks away, it does not mean that this price will no longer influence his or her judgments. The similarity of the extreme-priced product to the target, the recency of exposure to the extreme referent, and the situational and temporal contiguity of its presentation relative to the target can all influence how much the consumer will be willing to pay for the target. 
Consistent with our theorizing, we find in all three experiments that higher levels of relatedness between the high-priced referent and target lead to increases in reservation price. We also find in Experiments 1 and 2 that the presence of an extreme cue leads to greater changes in target reservation price when the extreme-priced referent and target are contiguously presented and the price information is readily accessible during judgment of the target. Mediation analysis in Experiments 1 and 2 shows that cue accessibility mediates the impact that target-extreme cue contiguity has on target reservation price. Results from the three experiments also show that these two effects are interdependent. The studies together suggest that the effect of an extreme cue's relatedness on reservation price tends to be greater when it is presented in close proximity to the target than when it is not.

Results from Experiments 2 and 3 further show that the hypothesized effects might be contingent on the nature of the product description. It is worth noting that nonbinary features are inherently ambiguous and therefore consumers are less likely to be knowledgeable about them. This lack of knowledge may make them more prone to the biasing influence of other extreme-priced products. Thus, in Experiment 2, we found support for all three hypotheses. On the other hand, when the product features are described in terms of binary features (Experiment 3), the extreme-priced product and the target seem less related and they have to occur in close proximity (high contiguity) before the extreme-priced product can exert an influence.

The results we obtained are obviously based on the type of stimuli we constructed. Although we attempted to make the experimental conditions as close to catalog shopping as possible, the stimuli we presented did not have brand names and the target products did not have prices. Thus, our procedure could be questioned on grounds of external validity. However, prior perceptions associated with known brand names made us choose an option that was more internally valid. Similarly, we could not have elicited a reservation price had we provided a price. Despite these limitations, the results we obtained suggested that there are several intriguing theoretical issues to consider.

For example, our results need to be considered in the context of other work in this area. Adaval and Monroe (2002) found that context prices had a contrast effect on judgments, and these types of effects were observed even when prices were presented subliminally. The results documented here, however, suggest assimilation effects in which the target's price moves in the direction of the extreme-priced referent. It is worth noting that the effects observed by Adaval and Monroe were on subjective judgments of cost (i.e., how expensive a product was perceived to be) and the impact this judgment had on price perception over time. The dependent measures we were concerned with in this article are reservation prices (the price people are willing to pay). Further research needs to examine how the subjective judgments of cost examined by Adaval and Monroe and the reservation price estimates discussed in our article are related and the conditions in which they guide behavior.

In addition, several other issues are in need of further inquiry. In our studies, participants were presented with little or no product category heterogeneity within a catalog. Given that catalogs generally contain products from multiple product categories, future studies should explore the effects of extreme-priced products in catalogs with a more varied product mix. In addition to product heterogeneity, it will be useful to examine what implications extremely low prices have on reservation prices. Because the "high" price has no limit, whereas the low price can be a minimum of zero, the potential effect of extremely low- versus extremely high-priced products is clearly not symmetric and needs to be explored.

Future work might also be extended to online Web environments. Online auction searches do not always produce directly relevant product offerings. Thus, the degree of relatedness among the products immediately surrounding the target product could be manipulated to determine if bids for unrelated products affect individual bids for target products. Additionally, the studies here are based on an extreme price of 10 times the average price for a given product. Future studies may examine the effects of differing levels of extreme-priced product cues.

The need for future work notwithstanding, our results indicate that the mere presence of extreme cues in catalogs can increase consumers' category reservation prices for other product categories in the catalog whether such products are from the same, related, or unrelated product categories. In addition, the positive effect of a strongly related extreme cue on reservation price even in low contiguity conditions suggests that extreme prices in catalogs may increase reservation price for same category products in stores bearing the name of the catalog company. Thus, if consumers see an extreme cue in a catalog and later go to a store of this catalog company (e.g., William Sonoma, J. Crew, Talbots), then they may be more inclined to buy a more expensive product. The good news for the consumer is that by putting a $\$ 1,000$ bread maker alongside a $\$ 100$ bread maker with few common features, the marketer is not very likely to impact consumer's reservation price for the latter. On the other hand, by including a $\$ 1,000$ bread maker in a catalog, the marketer can increase reservation price for the bread maker product category as a whole and also for other product categories such as food processors or pasta steamers.

Of course, marketers may intend extreme-priced products as just attention-getting gimmicks. However, our research shows that although gimmicky, the extreme item may make normally priced items look more reasonably priced. Even if unintentional, and consumers reject the extreme-priced product, what is interesting and relevant to policy is that the exposure may (consciously or unconsciously) raise reservation prices and willingness to pay. As such, subsequent search behavior may also be reduced - the logic being that if prices are 
misleadingly perceived as attractive due to the presence of an extreme price, the search might be prematurely truncated.

Given these implications, the results of this article highlight a potential public policy concern. If an extremely high-priced product increases reservation price, marketers may manipulate consumers into buying more expensive products and spending more money merely by including extreme-priced cues in their catalogs. If these extreme-priced product cues have little chance of being sold, then this practice could be viewed as deceptive marketing. Furthermore, because extreme cues seem to influence reservation prices of many products in various settings, this may be a more pervasive phenomenon than previously realized. Given that the choice of having extremely high-priced products in catalogs or stores lies entirely with marketers, such a practice may pose a potentially serious concern for consumers.

\section{ACKNOWLEDGMENTS}

This project was partially supported by grant HKUST$6192 / 04 \mathrm{H}$ awarded by the Research Grants Council, Hong Kong. We wish to thank Zeynep Gurhan-Canli and Joan Meyers- Levy for their suggestions on the paper. We also thank Phaythoune Chothmounethinh, Alexis Kronhaus, and Marissa Megge for providing research assistance.

\section{REFERENCES}

Adaval, R., \& Monroe, K. B. (2002). Automatic construction and use of contextual information for product and price evaluations. Journal of Consumer Research, 28, 572-588.

Ahluwalia, R., \& Gurhan-Canli, Z. (2000). The effects of extensions on the family brand name: An accessibility-diagnosticity perspective. Journal of Consumer Research, 27, 371-381.

Baron, R. M., \& Kenny, D. A. (1986). The moderator-mediator variable distinction in social psychological research: Conceptual, strategic and statistical considerations. Journal of Personality and Social Psychology, 51. 1173-1182.

Biswas, A. (1992). The moderating role of brand familiarity in reference price perceptions. Journal of Business Research, 25, 251-262.

Biswas A., \& Blair, E. A. (1991). Contextual effects of reference prices in retail advertisements. Journal of Marketing, 55, 1-12.

Chapman, G. B., \& Johnson, E. J. (1994). The limits of anchoring. Journal of Behavioral Decision Making, 7, 223-242.

Chapman, G. B., \& Johnson, E. J. (2003). Incorporating the irrelevant: Anchors in judgments of belief and value. In T. Gilovich, D. Griffin, \& D. Kahneman (Eds.), Heuristics and biases: The psychology of intuitive judgment (pp. 120-149). Cambridge, MA: Cambridge University Press.

Collins, A. M.., \& Loftus, E. F. (1975). A spreading-activation theory of semantic processing. Psychological Review, 82, 407-428.
Feldman, J. M., \& Lynch, J. G. (1988). Self-generated validity and other effects of measurement on belief, attitude, intention, and behavior. Journal of Applied Psychology, 73, 421-435.

Gillund, D., \& Shiffrin, R. M. (1984). A retrieval model for both recognition and recall. Psychological Review, 91, 1-67.

Higgins, E. T. (1996). Knowledge activation: Accessibility, applicability, and salience. In E. T. Higgins \& A. W. Kruglanski (Eds.), Social psychology: A handbook of basic principles (pp. 133-168). New York: Guilford.

Higgins, E. T., Rholes, W. S., \& Jones, C. R. (1977). Category accessibility and impression formation. Journal of Experimental Social Psychology, 13, 141-154.

Jacowitz, K. E., \& Kahneman, D. (1995). Measures of anchoring in estimation tasks. Personality and Social Psychology Bulletin, 21, 1161-1167.

Keller, K. L. (1998). Strategic brand management: Building, measuring, and managing brand equity. Upper Saddle River, NJ: Prentice Hall.

Krishna, A., Briesch, R., Lehmann, D., \& Yuan, H. (2002). A meta-analysis of the effect of price presentation on perceived savings. Journal of Retailing, 78, 101-118.

Lichtenstein, D. R., \& Bearden, W. O. ( 1989). Contextual influences on perceptions of merchant-supplied reference prices. Journal of Consumer Research, 15, 55-66.

Low, G. S., \& Lichtenstein, D. R. (1993). The effect of double deals on consumer attitudes. Journal of Retailing, 69, 453-466.

Mussweiler, T. M., \& Strack, F. (1999). Hypothesis-consistent testing and semantic priming in the anchoring paradigm: A selective accessibility model. Journal of Experimental Social Psychology, 35, 136-164.

Mussweiler, T. M., \& Strack, F. (2000). The use of category and exemplar knowledge in the solution of anchoring tasks. Journal of Personality and Social Psychology, 78, 1038-1052.

Nunes, J. C., \& Boatwright, P. (2004). Incidental prices and their effect on willingness to pay. Journal of Marketing Research, 41, 457-466.

Schkade, D. A., \& Johnson, E. J. (1989). Cognitive processes in preference reversals. Organizational Behavior and Human Decision Processes, 44, 203-231.

Strack, F., \& Mussweiler, T. M. (1997), Explaining the enigmatic anchoring effect: Mechanisms of selective accessibility. Journal of Personality and Social Psychology, 73, 437-446.

Taylor, V. A., \& Bearden, W. O. (2002). The effects of price on brand extension evaluations: The moderating role of extension similarity. Journal of the Academy of Marketing Science, 30, 131-140.

Tversky, A. (1977). Features of similarity. Psychological Review, 84, 327-352.

Tversky, A., \& Kahneman, D. (1974). Judgment under uncertainty: Heuristics and biases. Science, 185, 1124-1130.

Urbany, J. E., Bearden, W. O., \& Weilbaker, D. C. (1988). The effect of plausible and exaggerated reference prices on consumer perceptions and price search. Journal of Consumer Research, 15, 95-110.

Winer, B. J. (1971). Statistical principles in experimental design (2nd ed.). New York: McGraw-Hill.

Wyer, R. S. (2004). Social comprehension and judgment: The role of situation models, narratives, and implicit theories. Hillsdale, NJ: Lawrence Erlbaum Associates, Inc.

Wyer, R. S., \& Srull, T. K. (1989). Memory and cognition in its social context. Hillsdale, NJ: Lawrence Erlbaum Associates, Inc.

Received: May 26, 2005

Revision received: August 30, 2005

Accepted: September 22, 2005 